\title{
Development of Eating Support System Using Video Images: Investigation of Changes in Swallowing Function under Self-image Presentation
}

\author{
Kengo Hoyano $^{1 *}$ and Yasuhiro Ogoshi ${ }^{2}$ \\ ${ }^{1}$ Faculty of Health Science, Department of Rehabilitation, Division of Speech-Language-Hearing Therapy, \\ Fukui Health Science University, 55 Egami-cho 13-1, Fukui 910-3190, Japan \\ ${ }^{2}$ Graduate School of Engineering, University of Fukui, 3-9-1 Bunkyo, Fukui 910-8507, Japan
}

(Received March 31, 2020; accepted November 2, 2020)

Keywords: social facilitation, swallowing function, electromyography (EMG), electroencephalography (EEG)

In this study, we examined the effects of social facilitation by self-image presentation on muscle and brain activities during the intake of a modified diet for dysphagia. Under the selfand wall-image conditions, participants ingested gelled green tea, and electromyography (EMG) and electroencephalography (EEG) of the suprahyoid muscles were carried out before and after swallowing. EMG during swallowing showed significantly higher activity under the self-image condition. There was no significant difference in the activities of alpha and beta waves in the EEG before swallowing started. After the start of swallowing, the EEG showed a significant decrease in alpha wave activity under the self-image condition. The beta wave activity was significantly higher under the self-image condition and the alpha wave activity was significantly higher under the wall-image condition. These results showed that the activities of swallowingrelated muscles and the brain were altered after ingesting a modified diet for dysphagia during self-imaging.

\section{Introduction}

Many factors are involved in human eating behavior. The surrounding environment is considered to be one of the factors affecting eating behavior. Eating together is an environmental factor related to eating behavior. ${ }^{(1)}$ Previous studies have shown that eating with others increases food intake. ${ }^{(2)}$ De Castro reported an average increase in food intake of $40-50 \%$ when eating with someone compared with that when eating alone. ${ }^{(3)}$ Lumeng and Hillman also reported an increase in dietary intake in infants in a large group. ${ }^{(4)}$ Berry et al. reported that ice cream consumption was higher in a large group than in a small group, and larger in a small group than for an individual. ${ }^{(5)}$ Social facilitation was cited as the reason for the increase in intake in these studies. Social facilitation is a phenomenon in which an individual's behavior is affected by the presence of others. ${ }^{(6,7)}$ It has also been reported to

*Corresponding author: e-mail: k.hoyano@fukui-hsu.ac.jp

https://doi.org/10.18494/SAM.2020.2895 
promote eating, ${ }^{(8-11)}$ which is called the social facilitation of eating. The social facilitation of eating requires the presence of others. For example, chocolate was found to be more palatable and flavorful when consumed together with strangers even without communication. ${ }^{(12)}$ On the other hand, results suggesting that a self-image can promote food intake have also been reported. Nakata and Kawai carried out an experiment in which people ate popcorn in front of a mirror and also when a wall was reflected in the mirror. The results showed that people who ate popcorn with their own image had a higher consumption rate and a higher preference for popcorn. ${ }^{(13)}$ We have reported that a self-image condition on a monitor improved the intake rate of a modified diet for dysphagia and the subjective evaluation of food compared with a wallimage condition. ${ }^{(14)}$ From this finding, it is considered that the presence of others is not always necessary for the social facilitation of food.

If dysphagia is present, it can often be rehabilitated. Rehabilitation for dysphagia can be roughly divided into two types: indirect training without food and direct training with food. ${ }^{(15)}$ Indirect training is essentially safe and can be done for patients with severe dysphagia. Direct training involves the actual ingestion of food, and because there is a risk of aspiration, the form, posture, mouthful volume, and method of swallowing food are modified. In hospitals and facilities for the elderly, it has been reported that a modified diet for dysphagia is provided to an average of $35 \%$ of patients. ${ }^{(16)}$ This means that many patients with dysphagia eat other types of food during hospitalization, i.e., a modified diet for dysphagia that is safe and easy to swallow. A modified diet for dysphagia refers to a diet adjusted for shape, thickness, and ease of aggregation of the food bolus according to the level of swallowing function when the swallowing function is impaired, such as during swallowing and chewing. ${ }^{(17)}$ Morphological adjustments, such as pasting, are used to facilitate swallowing. However, it has been reported that the eating rate is lower in patients provided with pastelike foods in hospitals, and as a result, the intake of all nutrients, including energy, protein, lipids, carbohydrates, and vitamins, is below the dietary intake standard, the intake of vitamins and minerals is only half of the standard intake, and the body mass index (BMI) and serum albumin level in these patients are significantly lower than those in elderly people without dysphagia. ${ }^{(18)}$ In another report, a significant decrease in nutritional intake and skeletal muscle mass was observed in a group of patients who ate food prepared for swallowing. ${ }^{(19)}$ Thus, patients with dysphagia need to take food prepared for swallowing from the viewpoint of safety, but a decrease in food intake is a problem. One of the reasons for this is that the diet prepared for swallowing is different from the normal diet, and the appetite is decreased owing to the effect of the appearance, texture, and taste of the food. These factors may reduce the effectiveness of rehabilitation for dysphagia.

It is known that the eating environment changes the taste and intake of food in humans. King et al. reported that a friendly atmosphere with friends and family improved the evaluation of the tastiness of food. ${ }^{(20)}$ De Castro and Brewer also reported that the presence of friends and family increased food intake. ${ }^{(21)}$ Thus, it is considered that the presence of others affects the eating behavior of humans. Factors that influence eating behavior in the presence of others include the effects of the social facilitation of eating. ${ }^{(2)}$ De Castro and de Castro reported increased carbohydrate, fat, protein, and total caloric intake when eating with others. ${ }^{(23)}$ Bellisle and Dalix reported that food tastes better in the presence of others. ${ }^{(24)}$ These findings are considered to be due to the social facilitation of eating. 
It is possible that the presence of others has a positive effect on eating behavior, but in actual clinical practice, it is not always easy to set up an environment in which people can eat with others owing to the type of facility and staffing level. A previous study showed that the social facilitation of eating was achieved by presenting a self-image. ${ }^{(13)}$ We also showed improved subjective swallowability when healthy subjects ingested a modified diet for dysphagia under conditions in which they were shown a mirror image of themselves. ${ }^{(14)}$ These results suggest that self-image presentation may be effective in the social facilitation of eating, and a self-image may be easy to use as a stimulus in clinical practice. In this study, we measured the activities of the brain and suprahyoid muscles when a self-image was presented and a diet modified for swallowing was taken, and we examined the effect of the social facilitation of eating.

\section{Materials and Methods}

\subsection{Participants}

Twelve healthy adults (seven females and five males, age 19-25 years with a mean age of 20.91 years) participated in this study. They had no special dietary restrictions, food allergies, or medical history of eating disorders.

\subsection{Procedure}

The participants performed the experiment three hours after lunch. It was confirmed in a questionnaire that they were neither hungry nor full. The participants were instructed to take gelatinous green tea after an examiner presented a cue.

The participants were instructed to consume three tablespoons of the gelatinous green tea and to swallow it without chewing as much as possible. They were asked to sit on a chair with a desk in front of them. A 22-inch LED monitor (Dell Co., Ltd.; P 2213) was placed on the desk, and a web camera (ELECOM Co., Ltd.; UCAM-C0220FBNBK) was installed on the monitor. Their distance from the monitor was about $50 \mathrm{~cm}$. There were two conditions: the self-image condition, in which the web camera captured the participant's image and showed it on the monitor, and the wall-image condition, in which the web camera captured the wall behind the participant. Under the self-image condition, the web camera was adjusted so that the participant's upper body could be seen on the monitor. The wall-image condition was set as a control condition by using the same image as the participant's back.

Gelatinous green tea was presented to the participants. Green tea (Suntory Co., Ltd.; Iemon, $100 \mathrm{ml}$ ) was gelled with $3 \mathrm{~g}$ of a gelling agent (Healthy Foods Co., Ltd.; Toromi Smile). The gelatinous green tea $(10 \mathrm{ml})$ was placed in a plastic spoon $(5.5 \mathrm{~cm}$ long, $3.5 \mathrm{~cm}$ deep), and three tablespoons were filled with gelatinous green tea and placed on a stainless-steel tray.

Electromyography (EMG) was carried out to measure the activity of the suprahyoid muscles, and electroencephalography (EEG) was carried out to measure brain activity. A single board computer for biosignals (PLUX Co., Ltd.; BITalino (r)evolution) was used for EMG and EEG. A PLUX_EL-GEL-DISPOSABLE-24-25 electrode was used for EMG and EEG. EMG electrodes were attached to the anterior third of the left and right genio-mandibular areas. ${ }^{(25)}$ 
The EEG electrodes were affixed near Fpz in the 10-20 international electrode system. EMG and EEG data were recorded on a PC connected to the BITalino (r)evolution and Bluetooth using software for real-time biosignal visualization (PLUX Co., Ltd.; Opensignals) at a sampling rate of $1000 \mathrm{~Hz}$ (Fig. 1). The EEG and EMG were carried out after the intake of three tablespoons of gelatinous green tea under each condition (Fig. 2).

The recorded EMG activities were processed using the root mean square (RMS) after the processing of the absolute values. The range of analysis was defined as the mean $\pm 2 \mathrm{SD}$ of the resting muscle action potential at the onset of muscle action and at intervals of $400 \mathrm{~ms}$ thereafter. The EEG activities were determined by using the onset of EMG activity in the suprahyoid muscle as the base point for the start of the swallowing motion. EEG activities were extracted $1000 \mathrm{~ms}$ before the start of swallowing and $500 \mathrm{~ms}$ after the start of swallowing (Fig. 3). The EEG results were subjected to a fast Fourier transform into power spectra of alpha $(8-13 \mathrm{~Hz})$ and beta $(14-30 \mathrm{~Hz})$ waves.

\subsection{Statistical analyses}

The mean RMS values under the self-image and wall-image conditions and the power values of alpha and beta waves before and after the start of swallowing were evaluated by the paired $t$-test. The significance level was 5\%. Statistical analyses were performed using SPSS Statistics 25 (IBM Co., Ltd.).

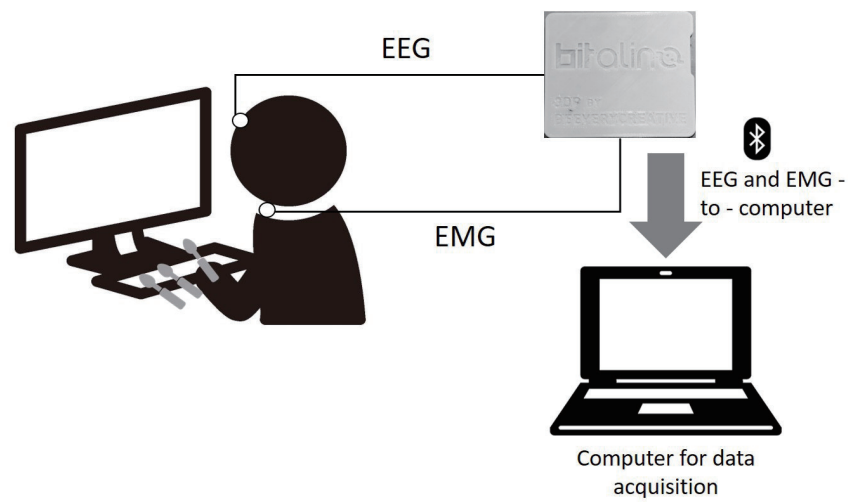

Fig. 1. EMG and EEG data recording methods.

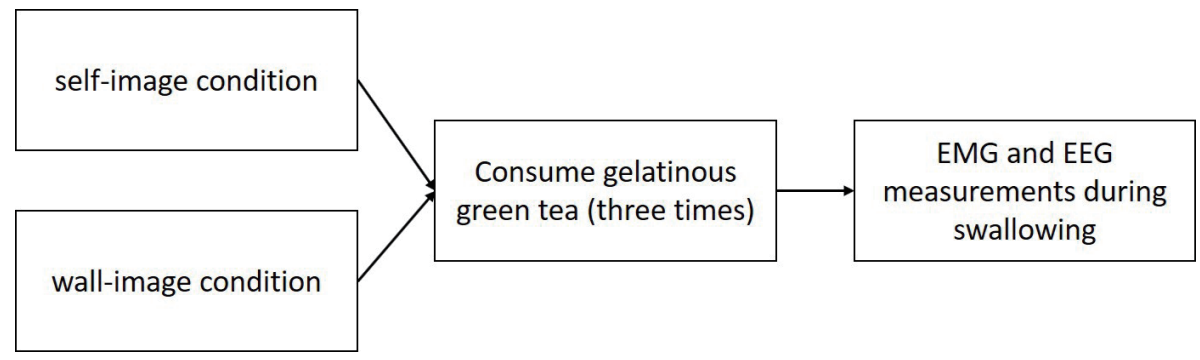

Fig. 2. Experimental procedure. 


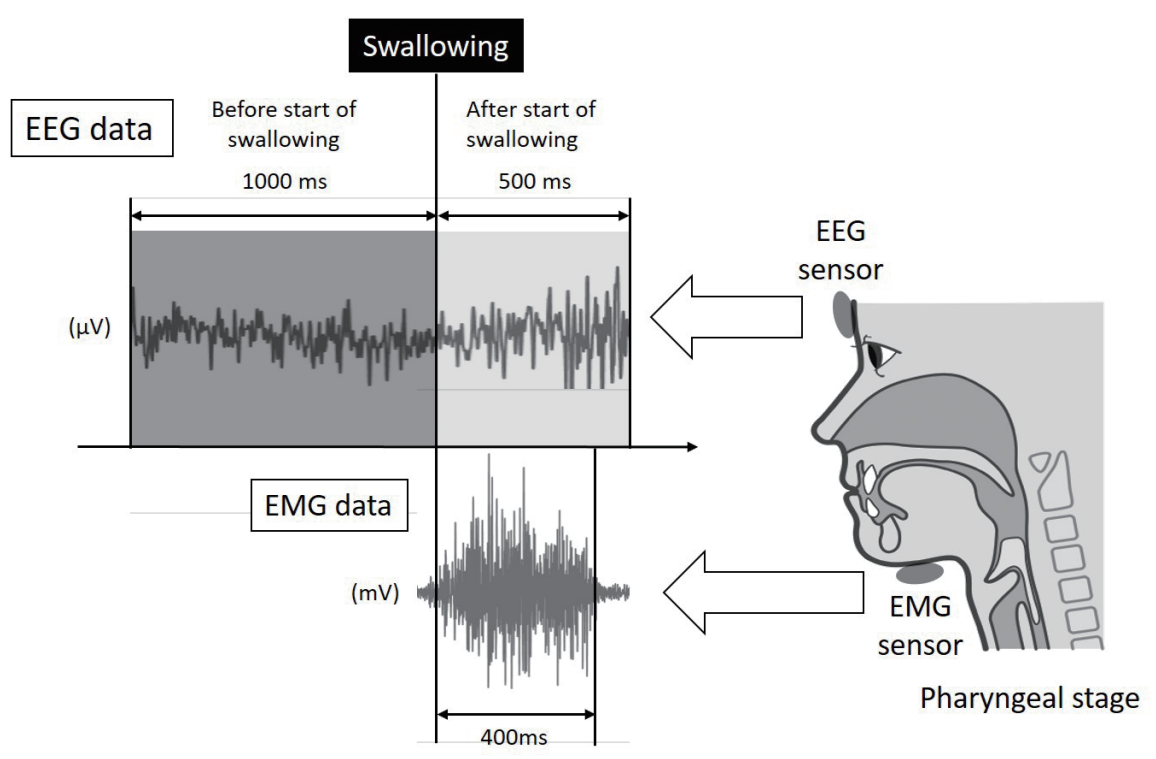

Fig. 3. EEG and EMG data.

\section{Results}

\subsection{EMG}

The EMG showed an RMS value of $0.072 \mathrm{mV}$ [standard error $(S E)=0.004$ ] under the selfimage condition and a value of $0.061 \mathrm{mV}$ under the wall-image condition $[S E=0.003][t(11)=3.512$, $p=0.005, d=0.753$ ] (Fig. 4).

\subsection{EEG}

The results of EEG before swallowing showed no significant difference in activity between the self-image condition [alpha waves: mean $(M)=4.882, S E=0.079$; beta waves: $M=4.774$, $S E=0.076$ ] and the wall-image condition [alpha waves: $M=4.845, S E=0.120$; beta waves: $M=4.795, S E=0.086$ ] for both alpha and beta waves [alpha waves: $t(11)=0.320, p=0.755$, $d=0.106$; beta waves: $t(11)=-0.193, p=0.850, d=0.072$ ] (Fig. 5). The self-image condition $[t(11)=1.026, p=0.327, d=0.404]$ and wall-image condition $[t(11)=0.557, p=0.589, d=0.142]$ showed no significant difference between alpha wave and beta wave activities (Fig. 6).

For alpha waves, the EEG showed that after swallowing, the power was significantly lower under the self-image condition $[M=4.400, S E=0.120]$ than under the wall-image condition $[M=4.734, S E=0.098][t(11)=-5.636, p=0.0001, d=0.888]$. For beta waves $[t(11)=3.558$, $p=0.004, d=0.547]$, the power was significantly higher under the self-image condition $[M=4.565$, $S E=0.108]$ than under the wall-image condition $[M=4.351, S E=0.119]$ (Fig. 7). Under the self-image condition, the power of beta waves was significantly increased $[t(11)=-2.453, p=0.032$, $d=0.419]$. Under the wall-image condition, the power of alpha waves was significantly increased $[t(11)=4.070, p=0.002, d=1.022]$ (Fig. 8). 


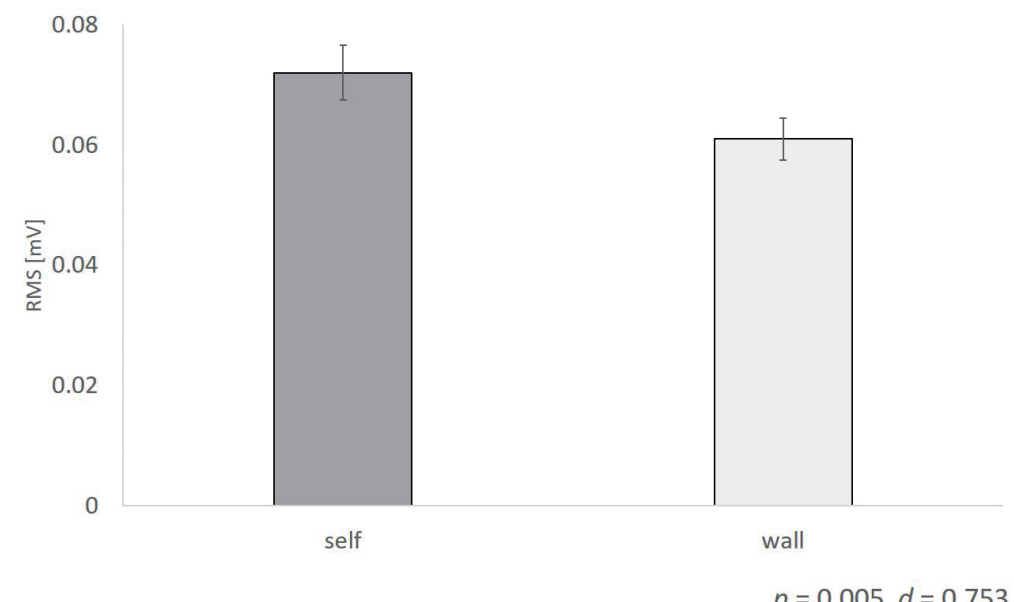

Fig. 4. EMG (RMS) of the suprahyoid muscles. The mean RMS under the self-image and wall-image conditions is shown. Error bars indicate average $\pm S E$.
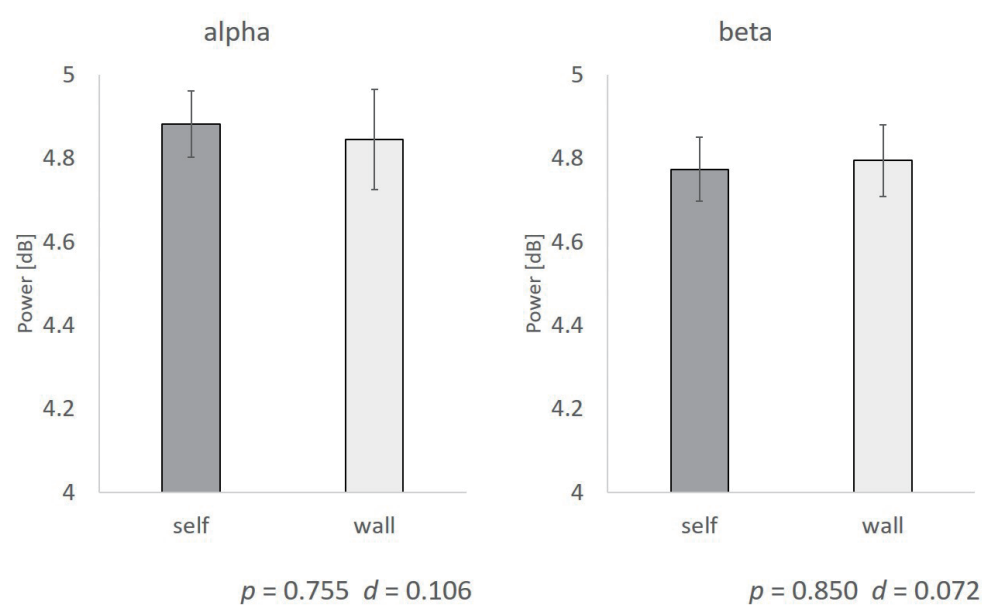

Fig. 5. EEG [power (dB)] under conditions before the start of swallowing. The mean of the logarithm of alpha and beta waves under the self-image and wall-image conditions is shown. Error bars indicate average $\pm S E$.
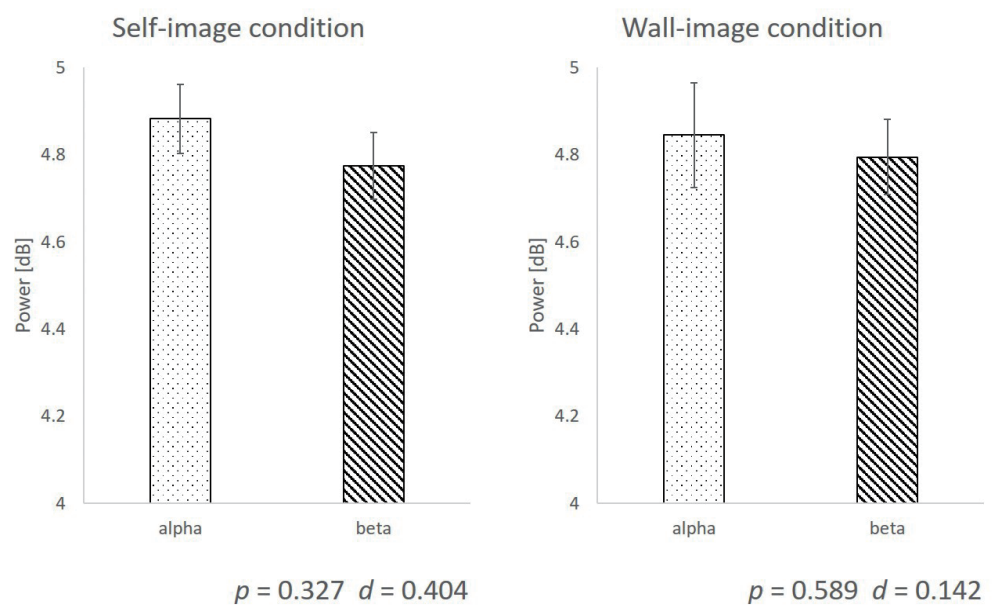

Fig. 6. Frequencies $1000 \mathrm{~ms}$ before the start of swallowing [power (dB)]. The mean of the logarithm of alpha and beta waves under the self-image and wall-image conditions is shown. Error bars indicate average $\pm S E$. 

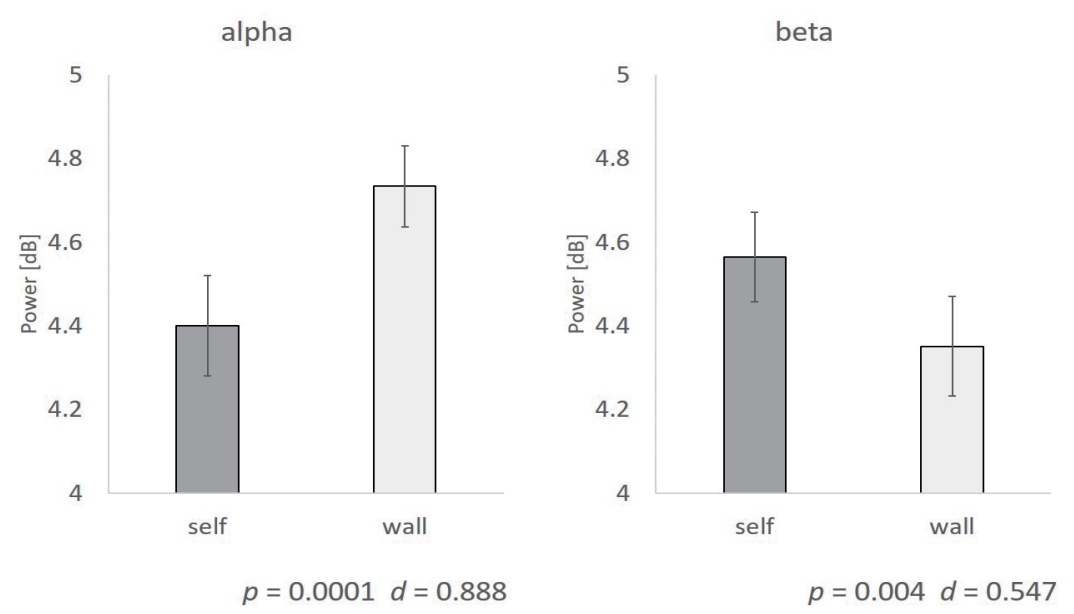

Fig. 7. EEG [power (dB)] $500 \mathrm{~ms}$ after the start of swallowing. The mean of the logarithm of alpha and beta waves under the self-image and wall-image conditions is shown. Error bars indicate average $\pm S E$.
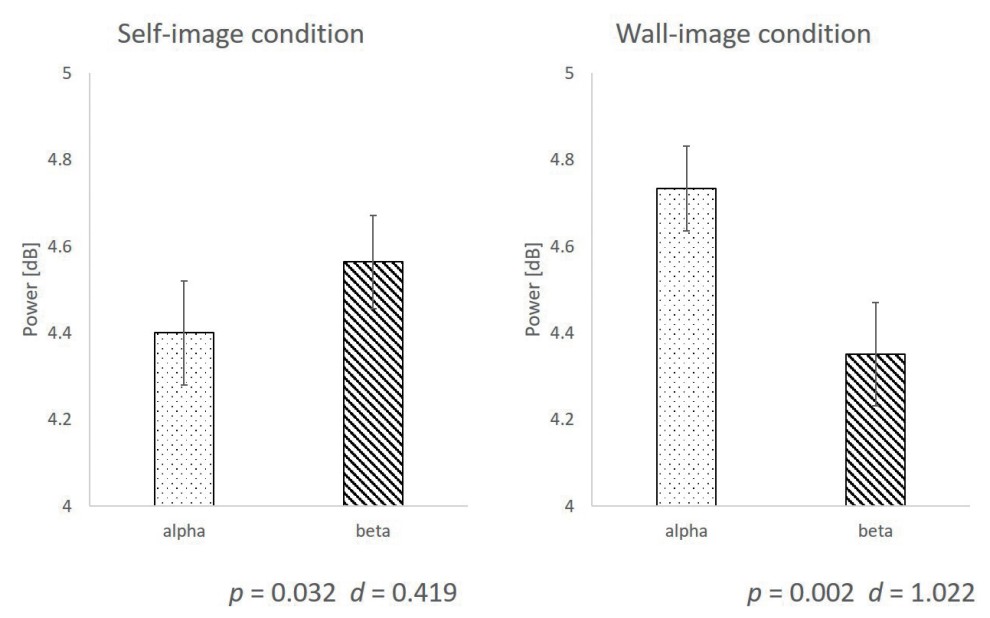

Fig. 8. Frequencies $500 \mathrm{~ms}$ after the start of swallowing [power (dB)]. The mean of the logarithm of alpha and beta waves under the self-image and wall-image conditions is shown. Error bars indicate average $\pm S E$.

\section{Discussion}

In the EMG of the suprahyoid muscles during swallowing, there was a significant difference in activity between the self-image condition and the wall-image condition, and a moderate effect was observed. Reynaud et al. reported that when rhesus monkeys performed simple tasks, their performance was improved simply by the presence of other animals. ${ }^{(26)}$ Chapman reported that muscle activity was significantly higher in the presence of others than that without the presence of others. ${ }^{(27)}$ In addition, it has been reported that the activity of the mylohyoid muscle, which is one of the suprahyoid muscles, increased significantly when normal children were observing others eating. ${ }^{(28)}$ The present results suggest that the presence of a human being 
sensed during the presentation of self-images may have caused a change in the activity of the suprahyoid muscles during swallowing. The activity of the suprahyoid muscles was increased during swallowing under the self-image condition, suggesting that there was an alteration in the swallowing function. However, the present study is based on self-images, and the results do not necessarily agree with those of previous studies in which other persons were present; thus, further investigation is necessary.

The EEG before swallowing showed no significant difference in activity between alpha and beta waves. For the present participants, since the instruction to watch the monitor was not given, it is possible that each participant was looking elsewhere. Moreover, the gaze easily deviated from the monitor during food intake, such as while holding the spoon, and the possibility that there was no difference in the brain activity was considered.

For alpha waves, the self-image condition decreased their activity significantly compared with that for the wall-image condition, and for beta waves, the self-image condition showed significantly increased activity compared with that for the wall-image condition. Liu et al. observed a decrease in the activity of alpha waves under social facilitation with two staff members compared with that for only one staff member working alone. ${ }^{(29)}$ Swallowing while distracted has been reported to reduce the activity of alpha waves compared with that while concentrating. ${ }^{(30)}$ A hypothesis of the social facilitation is the attention conflict theory. ${ }^{(31,32)}$ The hypothesis is that the presence of other people is a distraction, causing a conflict of attention to the task, thereby increasing arousal levels. ${ }^{(33)}$ Our results suggest that social facilitation occurred under the self-image condition. Demolliens et al. measured monkey brain activity in the presence of other monkeys. The results showed that some neurons in the prefrontal cortex were activated by the presence of other monkeys. ${ }^{(34)}$ Liu et al. also reported that beta wave activity increased when two staff members worked together compared with that when only one staff member worked alone. In the present study, the EEG electrode was installed in Fpz, and the activities recorded seemed to reflect those in the prefrontal area. ${ }^{(29)}$ These results suggest that the increased activity of beta waves reflects social facilitation.

In the intra-conditional comparison, the activity of beta waves was significantly higher than that of alpha waves under the self-image condition, and the activity of alpha waves was significantly higher than that of beta waves under the wall-image condition. Shimizu et al. reported that beta wave activity is higher in the swallowing stage than in the pharyngeal stage. ${ }^{(35)}$ In the present experiment, the EEG activity recorded after the start of swallowing reflected the activity of the suprahyoid muscle group, which may reflect the activity at the time corresponding to the pharyngeal stage. Alpha waves are considered to be associated with the attentional function. ${ }^{(36)}$ Under the wall-image condition, the absence of a target stimulus to direct attention may have increased the activity of alpha waves.

The present study suggests that the representation of self-portraits may have a social facilitation effect. However, because it has been reported that social facilitation occurs in the presence of others, it is necessary to compare this effect with the response in the presence of others. It has also been reported that self-portraits do not necessarily have a social facilitation effect and may have an inhibitory effect, ${ }^{(37)}$ and it is possible that the effect of self-portraits differs depending on the body shape and gender. Nakata and Kawai suggested that the social 
facilitation effect depends on the context and motivation; ${ }^{(38)}$ thus, it cannot be concluded that self-portraits have a social facilitation effect. It is necessary to investigate the factors affecting social facilitation by self-images. Moreover, it is necessary to examine whether the same effect can be achieved in patients with dysphagia depending on the cause and severity of dysphagia, higher brain dysfunction, and the presence of dementia.

These results suggest that social facilitation induces changes in brain activity during swallowing and that the activity of the supraglottic muscle group during swallowing is higher under the self-image condition than under the wall-image condition, suggesting that social facilitation occurs during self-image presentation and that the activity of swallowing-related muscle groups is altered.

In the present study, we found that the swallowing function was improved even by presenting one's own image, suggesting that it can be used as a stimulus, and it is possible that the same effect can be obtained by projecting self-images using a head mount display (HMD) without using virtual reality (VR) or augmented reality (AR). In addition, the effect of eye movement may be lessened by the use of an HMD, and it may be possible to obtain a greater social facilitation effect than that with monitor presentation.

In the future, we will examine the changes in the swallowing function when stimuli are presented via an HMD and a monitor, as well as the changes in the swallowing function when stimuli are presented to participants of different genders and ages. In the future, we plan to develop a food support system using an HMD on the basis of these findings.

\section{Conclusion}

In this study, it was observed that the activities of the brain and suprahyoid muscles changed when a modified diet for dysphagia was taken with a self-image presented. These changes clarified that social facilitation by self-image presentation affected muscle and brain activities. The application of the results of this study to an eating support system with stimulation presentation by VR and AR using an HMD will be examined in the future.

\section{Acknowledgments}

We thank the students of the Department of Speech Language Hearing Therapy at Fukui Health Science University for their cooperation as examinees in this experiment.

\section{References}

1 S. Imada: The Psychology of Eating: Why We Eat What We Eat (Yuhikaku, Tokyo, 2005) p. 1 (in Japanese).

2 C. P. Herman, D. A. Roth, and J. Polivy: Phycol. Bull. 129 (2003) 873.

3 J. M. de Castro: Physiol. Behav. 47 (1990) 1129.

4 J. C. Lumeng and K. H. Hillman: Arch. Dis. Child 92 (2007) 384.

5 S. L. Berry, W. W. Beatty, and R. C. Klesges: Appetite 6 (1985) 41.

6 F. H. Allport: J. Exp. Psychol. 3 (1920) 159.

7 M. Isozaki: J. Exp. Soc. Psychol. 19 (1979) 49.

8 J. M. de Castro, J. McCormick, M. Pedersen, and S. M. Kreizman: Physiol. Behav. 38 (1986) 25. 
9 B. Wansink and S. B. Park: Food Qual. Preference 12 (2001) 69.

10 V. I. Clendensen, C. P. Herman and J. Polovy: Appetite 23 (1994) 1.

11 S. C. King, H. L. Meiselman, A. W. Hottenstein, T. M. Work, and V. Cronk: Food Qual. Preference 18 (2007) 58.

12 E. J. Boothby, M. S. Clark, and J. A. Bargh: Psychol. Sci. 25 (2014) 2209.

13 R. Nakata and N. Kawai: Physiol. Behav. 179 (2017) 23.

14 K. Hoyano and Y. Ogoshi: Sens. Mater. 30 (2018) 1517.

15 E. Saitoh and K. Ueda: Dysphagia Rehabilitation (Isiyaku, Tokyo, 2016) 3rd ed., p. 194 (in Japanese).

16 J. Kayashita, H. Ogoshi, H. Maeda, K. Takahashi, I. Fujishima, and J. Fujitani: J. J. Dysphagia. Rehabil. 15 (2001) 209 (in Japanese).

17 The Japan Foundation for Aging and Health: https://www.tyojyu.or.jp/net/byouki/engeseihaishikkan/engeshoku.html (accessed March 2020).

18 S. Tokunaga and N. Hiruta: Human Nutrition 6 (2014) 84 (in Japanese).

19 A. Shimizu, K. Maeda, K. Tanaka, M. Ogawa, and J. Kayashita: Geriatr. Gerontol. Int. 18 (2018) 698.

20 S. C. King, H. L. Meiselman, A. W. Hottenstein, T. M. Work, and V. Cronk. Food Qual. Preference 28 (2007) 58.

21 J. M. de Castro and E. M. Brewer: Physiol. Behav. 51 (1992) 121.

22 C. P. Herman: Appetite 86 (2015) 61.

23 J. M. de Castro and E.S. de Castro: Am. J. Clin. Nutr. 50 (1989) 237.

24 F. Bellisle and A. M. Dalix: Am. J. Clin. Nutr. 74 (2001) 197.

25 T. de Mayo, R. Miralles, D. Barrero, A. Bulboa, D. Carvajal, S. Valenzuela, and G. Ormeño: J. Oral Rehabil. 32 (2005) 487.

26 A. J. Reynaud, C. Guedj, F. Hadj-Bouziane, M. Meunier, and E. Monfardini: Front Behav. Neurosci. 9 (2015) 328.

27 A. J. Chapman: Br. J. Psychol. 65 (1974) 123.

28 L. Cattaneo, M. Fabbri-Destro, S. Boria, C. Pieraccini, A. Monti, G. Cossu, and G. Rizzolatti: Proc. Natl. Acad. Sci. USA 104 (2007) 17825.

29 N. Liu, R. Yu, L. Yang, and X. Lin: Sci. Rep. 7 (2017) 15073. https://doi.org/10.1038/s41598-017-15437-y.

30 I. Jestrovic, J. L. Coyle, S. Perera, and E. Sejdić: Brain Struct. Funct. 223 (2018) 955.

31 N. B. Cottrell, D. L. Wack, G. J. Sekerak, and R. H. Rittle: J. Pers. Soc. Psychol. 9 (1968) 245.

32 R. S. Baron: Advances in Experimental Social Psychology 19 (Academic Press, New York, 1986) p. 1.

33 T. Ikegami and Y. Endo: Graphic Social Psychology (Science, Tokyo, 2008) 2nd ed., p. 228 (in Japanese).

34 M. Demolliens, F. Isbaine, S. Takerkart, P. Huguet, and D. Boussaoud: Soc. Cogn. Affect Neurosci. 12 (2017) 1241.

35 N. Shimizu, A. Takahashi, A. Horino, and S. Yamada: Jpn. J. Nurs. Administration 28 (2018) 818 (in Japanese).

36 M. Vázquez, Marrufo, E. Vaquero, M. J. Cardoso, and C. M. Gómez: Brain Res. Cogn. Brain Res. 12 (2001) 315.

37 S. M. Sentyrz and B. J. Bushman: J. Appl. Psychol. 83 (1998) 944.

38 R. Nakata and N. Kawai: Cognit. Studies 26 (2019) 86 (in Japanese). 